\title{
Overgrowth syndromes: is dysfunctional PI3-kinase signalling a unifying mechanism?
}

\author{
Karen T Barker ${ }^{*}, 1$ and Richard S Houlston ${ }^{1}$ \\ ${ }^{1}$ Section of Cancer Genetics, Institute of Cancer Research, Surrey, SM2 5NG, UK
}

Studies in drosophila and animal models have shown that the phosphoinositide-3-kinase (PI3-kinase) axis plays a central role in normal development, defining the number and size of cells in tissues. Dysfunction of this pathway leads to growth anomalies and has been established to play a key role in the pathogenesis of Cowden syndrome and tuberous sclerosis. It is probable that dysfunction of this pathway is the basis of other disorders especially those typified by asymmetric overgrowth.

European Journal of Human Genetics (2003) 11, 665-670. doi:10.1038/sj.ejhg.5201026

Keywords: overgrowth; PI3-kinase; Cowden syndrome; tuberous sclerosis; Proteus syndrome

\section{Introduction}

A myriad of syndromes are characterised by substantial localised or asymmetric tissue overgrowth. Pre-eminent among these is the Proteus syndrome (PS) (MIM176920), a rare disorder characterised by postnatal overgrowth of multiple tissues derived from different cell lineages. ${ }^{1-3}$ The main diagnostic criteria for PS are that it is sporadic, mosaic and follows a progressive course. ${ }^{3}$ Several other overgrowth syndromes have overlapping features with PS, such as Klippel-Trenauney-Syndrome (KTS) (MIM149000), characterised by large cutaneous hemangiomata with hypertrophy of the related bones and soft tissues. Enlargement of limbs on one side of the body defines the syndrome of hemihypertrophy or hemihyperplasia (MIM235000). Overgrowth may, however, be more limited involving a single limb-simple hemihyperplasia, or overgrowth of one side of the face-hemifacial hyperplasia (MIM133900).

A common feature of all these disorders is that tissue overgrowth is asymmetric but paradoxically involves more than one cell lineage. This raises a number of questions about the nature of the molecular insult underpinning such disorders. In the case of PS at least, the hypothesis has been proposed that it is caused by a postzygotic somatic mutation, in a gene that is lethal in the nonmosaic state. ${ }^{4}$

*Correspondence: KT Barker, Section of Cancer Genetics, Institute of Cancer Research, Surrey SM2 5NG, UK. Tel: +44 (0) 208722 4252; Fax: +44 (0) 208722 4359; E-mail: karenb@icr.ac.uk

Received 13 January 2003; revised 28 March 2003; accepted 2 April 2003
Here we review the experimental data relating to cell, organ and tissue overgrowth, relate this to human overgrowth diseases and propose that PI3-kinase dysfunction is likely to be a common theme in this class of disorders.

\section{Role of PI3-kinase in control of tissue growth}

Apart from selectively bred species such as dogs, animals of the same species tend to be the same size. Within a species, organ and limb size are also fairly constant. Evidence from animal experiments supports the hypothesis of a 'Total Mass Checkpoint' - a mechanism for detecting and regulating organ size accordingly.,6 Tissue overgrowth represents a breakdown in the checkpoint mechanism underpinning control of overall size. This is a consequence of dysfunction in one or more processes - apoptosis, cellular proliferation and cell growth. In order for cells to grow (increase in mass), they must synthesise various macromolecules such as the structural components of the cell. This requires an increase in the translational apparatus including ribosomes and translation factors necessary for protein synthesis. The mRNAs that encode these are characterised by an oligopyrimidine tract at their $5^{\prime}$ translational start site (5'TOP mRNAs). The translation of $5^{\prime}$ TOP mRNAs is regulated by $40 \mathrm{~S}$ ribosomal protein $\mathrm{S} 6$ kinase(s) (S6K). ${ }^{7}$ In the absence of stimulation $\mathrm{S} 6 \mathrm{~K}$ is inactive. The activity of the kinase is regulated in response to both nutrient availability and insulin/insulinlike growth factor (IGF) signalling. ${ }^{7}$ Recent studies have 
shown that the link between the insulin/IGF signallingpathway and the growth of cells is through PI3-kinase (Figure 1). ${ }^{8-12}$

Insulin receptor stimulation leads to an increase in PI3kinase activity thereby generating PIP3, leading to the activation of $\mathrm{PKB} / \mathrm{Akt}^{13-16}$ The consequences of PKB/Akt activation include the phosphorylation and inhibition of glycogen synthase kinase 3 (GSK3), resulting in the dephosphorylation of substrates of GSK3, including glycogen synthase and eukaryotic initiation factor 2B (eIF2B). These contribute to the insulin-induced stimulation of glycogen and protein synthesis.

Recent studies have revealed the link between PI3-kinase stimulation and growth control through recognition of the functions of TSC1 and TSC2 (hamartin and tuberin, respectively). These were first identified in humans as the products of the genes mutated in patients with dominantly inherited tuberous sclerosis (MIM191100). TSC1 and TSC2 interact in a complex that inhibits the activity of S6K. ${ }^{8-12}$ Phosphorylation of TSC2 by $\mathrm{PKB} /$ Akt inhibits the function of the TSC1 and TSC2 complex, thus relieving the inhibition of S6K, and allowing translation of $5^{\prime} \mathrm{TOP}$ mRNA. Nutrient stimulation has been shown to activate S6K via the mammalian target of rapamycin (mTOR), causing the release of eukaryotic translation initiation

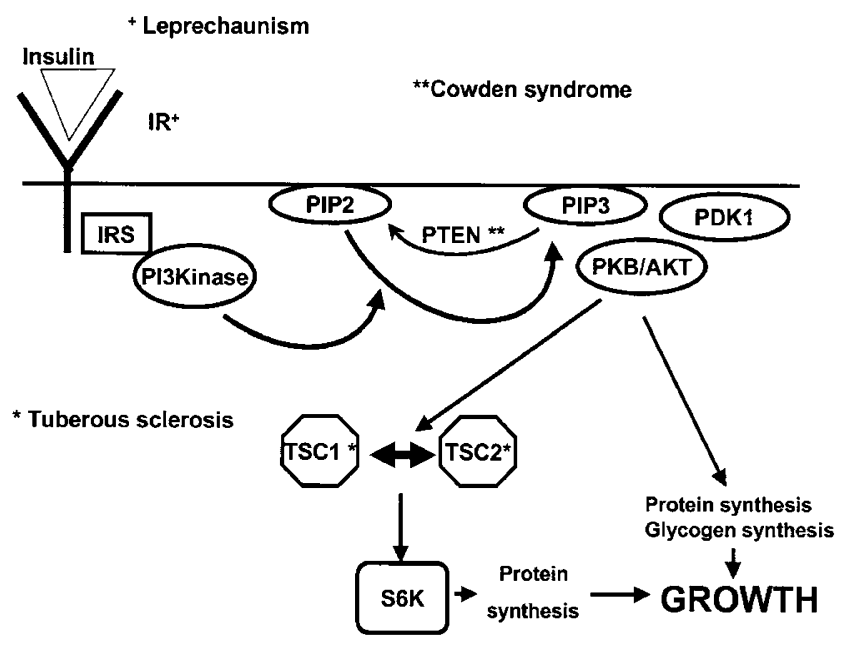

Figure 1 Model of the insulin/IGF signalling pathway via PI3-kinase: effects on cellular growth. Upon insulin binding the insulin receptor becomes phosphorylated on specific tyrosine residues, this leads to recruitment of IRS, and then PI3-kinase which catalyses the production of PIP3. PKB/Akt binds to PIP3 and is activated by PDK1. PKB/Akt has a number of effects, inhibition of GSK3 leads to glycogen and protein synthesis via glycogen synthase and eiF2B activation. PKB/Akt also phosphorylates TSC2 leading to functional disruption of the TSC1-TSC2 complex, relieving S6Kinase inhibition and resulting in mRNA translation. As described in the text Cowden syndrome, tuberous sclerosis and leprechaunism result from loss of PTEN, TSC1 or TSC2 and IR function, respectively. factor 4e (eIF4E) by phosphorylation of eIF4E binding protein (4E-BP1). This is a rate-limiting step of eukaryotic translation. ${ }^{17}$ There is evidence to suggest that the complex of TSC1-TSC2 antagonises S6K activation via mTOR. ${ }^{12}$

There is considerable data from animal models that disruption of the PI3-kinase pathway results in disordered growth. In drosophila, altering the activities of components of the insulin-signalling pathway have been shown to affect cell, organ, and overall size. Overexpression of drosophila insulin receptor (dinr), drosophila PI3-kinase (dp110) and drosophila PKB/Akt (dAkt) led to an increase in cell and organ size, whereas loss of function mutations in dinr, dp110, chico (drosophila IRS), dAkt and drosophila S6K (dS6K) led to a decrease in cell and organ size. ${ }^{18}$ Mutations in all components, except dS6K, also affect cell number. ${ }^{19}$ PTEN acts as a negative regulator of PI3-kinase signalling by catalysing the dephosphorylation of PIP3. ${ }^{20,21}$ Overexpression of drosophila PTEN (dpten) leads to reduced cell and organ size and conversely cells mutant for dpten are larger than wild type. ${ }^{22}$ Drosophila TSC1 and TSC2 (dTSC1 and dTSC2) function in concert to antagonise insulin signalling in regulating organ size, the effect appears to be downstream of dAkt. ${ }^{23,24}$

Cardiac expression of constitutively active PI3-kinase in transgenic mice has been shown to lead to cardiac enlargement, whereas dominant negative PI3-kinase results in smaller hearts. ${ }^{25}$ The change in heart size is a direct consequence of a change in cell size. ${ }^{25} \mathrm{PKB} / \mathrm{Akt}$ is an important downstream effector of PI3-kinase signaling. ${ }^{14}$ Similarly, expression of constitutively active PKB/Akt in the mouse heart leads to an increase in size, as a consequence of an increase in cell size. ${ }^{26}$

Neuronal-specific deletion of PTEN in murine brains results in the expression of high levels of phosphorylated $\mathrm{PKB} /$ Akt and an increase in cell body size without evidence of abnormal proliferation. ${ }^{27-29}$ Intriguingly these abnormalities closely resemble the histology of human LhermitteDuclos disease, a variant of Cowden disease caused by germline PTEN mutations. ${ }^{27-29}$

The Jurkat T-cell human leukaemic cell line does not express endogenous PTEN. Through the establishment of stable cell lines, transfected with PTEN in inducible vectors $\mathrm{Xu}$ et $\mathrm{al}^{30}$ have shown that PTEN decreases cell size via negative regulation of PI3-kinase signaling.

\section{Role of PI3-kinase dysfunction in human disease - Cowden disease and tuberous sclerosis - primary examples}

Although the data from drosophila and mice provides $a$ priori evidence to support a role in humans, the only way of definitively establishing this is by demonstrating that a specific lesion in the pathway influences phenotype or is disease causing. A number of diseases associated with disordered growth are now recognised to result from 
defects in the PI3-kinase axis (Table 1). Inactivating mutations in the insulin receptor gene cause leprechaunism. Cowden syndrome is caused by germline loss of function mutations in PTEN. PTEN functions to antagonise PI3-kinase signalling, thus when its activity is removed the pathway can proceed in an uncontrolled fashion. Tuberous sclerosis is caused by inactivating mutations in either TSC1 or TSC2. Inactivation of TSC1 or TSC2 leads to a failure of S6K inhibition resulting in unrestrained growth. Furthermore, tuberous sclerosis causing TSC2 mutations have in vitro been shown to reduce inhibition of S6K phosphorylation, suggesting that $\mathrm{S} 6 \mathrm{~K}$ regulation is biologically important. ${ }^{10-12}$

Lymphedema-distichiasis, which shares some features with other syndromes in this collective, such as lipomas, is caused by mutations in FOXC2 - a forkhead family transcription factor. FOXC2 regulates adipocyte metabolism, its expression being stimulated by insulin and TNF $\alpha$ via PI3-kinase and ERK1/2-dependent pathways. ${ }^{31}$ Sotos syndrome has recently been shown to be due to mutations in NSD1. ${ }^{32}$ It is intriguing to speculate that the effect of NSD1 dysfunction may be mediated through the PI3kinase pathway; however, to date, there is no evidence to support such a postulate.

\section{Discussion - PI3-kinase dysfunction in PS?}

There is increasing evidence indicating that the PI3-kinase pathway is central to normal cellular and organ development and that perturbation of this axis can result in disease in man. Disruption of the PI3-kinase pathway in both drosophila and mice has an effect on organ size in part due to an effect on cell size; in humans large neurones are a feature of Lhermitte-Duclos disease, ${ }^{33}$ and giant cell astrocytomas are commonly observed in tuberous sclerosis individuals. $^{34}$

A defining feature of PS is its clinical variability especially with respect to the distribution of lesions. This contrasts to Sotos syndrome, which is characterised by a generalised overgrowth of multiple cell types and Cowden disease where there is a distinct pattern of lesions. The tissue specificity in Cowden disease is characteristic of many inherited cancer predisposition syndromes, which show tissue-specific effects. This may in part be due to differential cell/tissue type susceptibilities to PTEN mutations.

One explanation for the distribution of lesions in PS has been proposed by Happle, ${ }^{4}$ in which a postzygotic mutation occurs early in development and that the cells bearing the mutation are randomly distributed. Depending upon the cell type harbouring the lesion, and the stage of development this occurred, different patterns of affected tissues would be seen.

PS and KTS are both presumed to be the result of somatic molecular defects, ${ }^{4}$ thus they are not amenable to identification by conventional strategies such as genetic linkage analysis. On the basis of available information, genes encoding components of the PI3-kinase pathway represent candidates for both diseases. Moreover there is already some evidence to support such a rationale for examining candidate genes within this pathway in asymmetric overgrowth disorders.

Table 1 Syndromes characterised by dysfunctional PI3-kinase signalling

\begin{tabular}{|c|c|c|c|c|}
\hline Syndrome & MIM & Gene mutated & Clinical features & Minor \\
\hline Leprechaunism & 246200 & $I R$ & $\begin{array}{l}\text { Intrauterine growth retardation } \\
\text { Elfin-like features }\end{array}$ & $\begin{array}{l}\text { Decreased subcutaneous fat } \\
\text { Hyertrichosis } \\
\text { Acanthosis nigricans }\end{array}$ \\
\hline Cowden & 158350 & PTEN & $\begin{array}{l}\text { Breast and thyroid cancer } \\
\text { Macrocephaly } \\
\text { Lhermitte-Duclos disease }\end{array}$ & $\begin{array}{l}\text { Benign thyroid disease } \\
\text { Lipomas } \\
\text { Fibromas } \\
\text { Gastrointestinal hamartomas } \\
\text { Genitourinary tumours }\end{array}$ \\
\hline Tuberous sclerosis & 191100 & TSC $1 / T S C 2$ & $\begin{array}{l}\text { Retinal hamartomas } \\
\text { Facial Angiofibromas } \\
\text { Renal angiomyolipomata } \\
\text { Rhabdomyoma } \\
\text { Bone cysts } \\
\text { Subependymal glial nodule }\end{array}$ & $\begin{array}{l}\text { Giant cell astrocytomas } \\
\text { Honeycomb fibrosis }\end{array}$ \\
\hline Lymphedema-distichiasis & 153400 & FOXC2 & $\begin{array}{l}\text { Lymphedema } \\
\text { Distichiasis }\end{array}$ & \\
\hline $\begin{array}{l}\text { Proteus and 'Proteus-like' } \\
\text { syndromes }\end{array}$ & 176920 & PTEN & $\begin{array}{l}\text { Vascular malformation } \\
\text { Lipomatosis } \\
\text { Hemihypertrophy }\end{array}$ & $\begin{array}{l}\text { Macrocephaly } \\
\text { Epidermoid naevi }\end{array}$ \\
\hline
\end{tabular}


Given the fundamental role of PI3-kinase signalling in the control of tissue growth, it is conceivable that a gain of function mutation in a positive regulator of the PI3-kinase pathway could cause PS. Similarly, homozygous disruption or knockout of PTEN (which in mice, has been shown to result in embryonic lethality) ${ }^{35,36}$ or another key regulator would equate to the same unrestrained PI3kinase signalling. Such a notion is totally in keeping with Happle's proposal that PS is caused by a postzygotic mutation that is lethal in the whole organism but can survive in the mosaic state. ${ }^{4}$

The first evidence that PI3-kinase signalling may be involved in the aetiology of PS comes from Rudolph et al, ${ }^{37}$ who reported a patient with reduced serum levels of IGFs IGF-I and especially IGF-II, and the major growth hormone-dependent binding protein (IGFBP-3). Fibroblasts cultured from affected tissue showed an unusual pattern of IGF binding proteins compared with normal fibroblasts, containing large amounts of an IGFBP with high affinity to IGF-II. They suggested that IGF production is generally disturbed in PS with an imbalance in levels of specific IGFBPs in regions of overgrowth. ${ }^{37}$ A role for IGFBP2 in the inhibition of the adrenocortical cell size has been shown through overexpression of the growth hormone $(\mathrm{GH})$ and IGFBP2, either alone or in combination, in transgenic mice. ${ }^{38}$ Although these data provide support for a role of PI3-kinase dysfunction in the aetiology of PS it is only indirect. The identification of inactivating PTEN mutations in a subset of PS provides direct evidence for such a role. Given the similarities between PS and Cowden disease, Zhou et al ${ }^{39,40}$ examined the PTEN gene for involvement in PS. De novo germline PTEN mutations have been detected in two individuals with PS and three patients with a 'proteus-like' syndrome (PS-like). A further case, with unequivocal PS, has recently been reported to harbour a truncating de novo germline PTEN mutation. ${ }^{41}$ In one PS-like patient reported by Zhou et $a l^{39,40}$ a 'second hit' mutation in the normal allele in affected tissues was demonstrated. However, in another PS-like case reported by Zhou et al $^{40}$ and the PS case reported by Smith et al, ${ }^{41}$ no second mutation in affected tissues has been detected. ${ }^{40,41}$ Whether or not haploinsufficiency of PTEN per se is able to cause this phenotype is unclear. The evidence for mosaicism in PS (epidermal lesions following lines of Blaschko) is perhaps more suggestive of PTEN being inactivated by an epigenetic mechanism or possibly mutation of another gene in the same pathway in the affected tissues. Although the role of PTEN in overgrowth in the context of PS may be restricted, as other workers have not replicated these findings, PS is likely to be highly heterogeneous. ${ }^{42,43}$ Hence, the data lends support to the notion that disruption of the PI3-kinase pathway may well be important outside the context of Cowden syndrome per se.

This leads to another possible explanation for the events leading to PS. It may be that PS requires two 'hits' and that

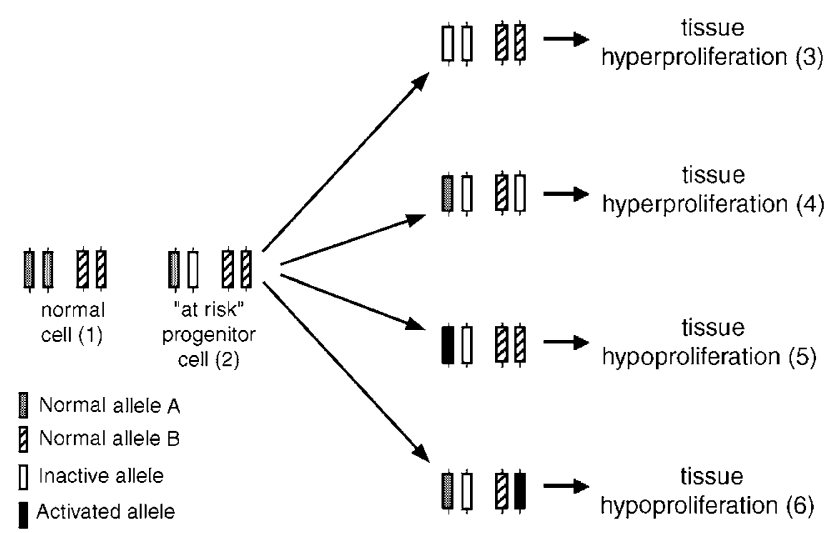

Figure 2 Two-hit model for the development of PS. Situation 1 corresponds to the normal cell. Inactivation of one allele of a negative regulatory gene $A$ within the growth control pathway generates the 'at risk' progenitor cell - state 2 . If the other allele of $A$ is subsequently inactivated this leads to unrestrained growth and tissue hyperproliferation - state 3 . Unrestrained growth could alternatively be a consequence of inactivation of another negative regulatory gene (B) in the same pathway - state 4 . If the other A allele undergoes an activation mutation then this would downregulate the growth pathway leading to tissue hypoproliferation - state 5 . hypoproliferation would also occur if gene B were activated state 6.

these may be in different genes that impact on the same pathway (Figure 2). An individual carrying a germline mutation in one gene may be predisposed to PS but the second 'rare' event is required for the development of the disease, as suggested in the case reported by Smith et al. ${ }^{41}$

Timing, location and nature of the second event may also impact on the severity of the lesion. This type of model can be further expanded to encompass the phenomenon of Elattoproteus described by Happle, ${ }^{44}$ where both regions of overgrowth and deficient growth have been described in some PS cases. It is feasible that in regions of deficient growth there was a second event that overcompensated for the initial lesion. Such a mechanism would be analogous to the phenotypes associated with mutations in FGFR3; here, an activating (gain of function) point mutation leads to achondroplasia (MIM100800), and mice homozygous for a targeted disruption of FGFR3 exhibit bone overgrowth. ${ }^{45}$ To fit with the hypothesis that dysregulation of the PI3-kinase pathway is responsible for overgrowth, inactivation of the pathway could cause deficient growth as supported by findings in drosphilla. Revertant mosaicism, the spontaneous correction of a deleterious mutation during mitosis, has been noted in some disorders. For example, in epidermolysis bullosa, caused by a deficiency of collagen $17 \alpha-1$, patches of normal skin, containing collagen are seen. ${ }^{46}$

Existing data provide strong evidence that the PI3-kinase pathway is critical to normal tissue and organ development and dysfunction is associated with diseases characterised 
by disordered growth. Furthermore, it is likely that knowledge of this pathway will provide a basis for understanding phenotypic similarities between overgrowth disorders and the relation between phenotype and genotype. At present the number of diseases caused by known defects is restricted, suggesting that others caused by mutations in genes encoding other components of the PI3-kinase pathway are likely to be identified.

\section{References}

1 Cohen Jr MM, Hayden PW: A newly recognized hamartomatous syndrome. Birth Defects Orig Artic Ser 1979; 15: 291-296.

2 Wiedemann HR, Burgio GR, Aldenhoff P, Kunze J, Kaufmann HJ, Schirg E: The proteus syndrome. Partial gigantism of the hands and/or feet, nevi, hemihypertrophy, subcutaneous tumors, macrocephaly or other skull anomalies and possible accelerated growth and visceral affections. Eur J Pediatr 1983; 140: $5-12$.

3 Biesecker LG, Happle R, Mulliken JB et al: Proteus syndrome: diagnostic criteria, differential diagnosis, and patient evaluation. Am J Med Genet 1999; 84: 389-395.

4 Happle R: Lethal genes surviving by mosaicism: a possible explanation for sporadic birth defects involving the skin. J Am Acad Dermatol 1987; 16: 899-906.

5 Stocker H, Hafen E: Genetic control of cell size. Curr Opin Genet Dev 2000; 10: 529-535.

6 Potter CJ, Xu T: Mechanisms of size control. Curr Opin Genet Dev 2001; 11: 279-286.

7 Dufner A, Thomas G: Ribosomal S6 kinase signaling and the control of translation. Exp Cell Res 1999; 253: 100-109.

8 Kwiatkowski DJ, Zhang H, Bandura JL et al: A mouse model of TSC1 reveals sex-dependent lethality from liver hemangiomas, and up-regulation of p70S6 kinase activity in Tsc1 null cells. Hum Mol Genet 2002; 11: 525-534.

9 Goncharova EA, Goncharov DA, Eszterhas A et al: Tuberin regulates p70 S6 kinase activation and ribosomal protein S6 phosphorylation. A role for the TSC2 tumor suppressor gene in pulmonary lymphangioleiomyomatosis (LAM). J Biol Chem 2002; 277: 30958-30967.

10 Potter CJ, Pedraza LG, Xu T: Akt regulates growth by directly phosphorylating Tsc2. Nat Cell Biol 2002; 4: 658-665.

11 Gao X, Zhang Y, Arrazola P et al: Tsc tumour suppressor proteins antagonize amino-acid-TOR signalling. Nat Cell Biol 2002; 4: 699704 .

12 Inoki K, Li Y, Zhu T, Wu J, Guan KL: TSC2 is phosphorylated and inhibited by Akt and suppresses mTOR signalling. Nat Cell Biol 2002; 4: 648-657.

13 Shepherd PR, Withers DJ, Siddle K: Phosphoinositide 3-kinase: the key switch mechanism in insulin signalling. Biochem J 1998; 333: 471-490.

14 Cantley LC: The phosphoinositide 3-kinase pathway. Science 2002; 296: 1655-1657.

15 Vanhaesebroeck B, Waterfield MD: Signaling by distinct classes of phosphoinositide 3-kinases. Exp Cell Res 1999; 253: 239-254.

16 Scheid MP, Marignani PA, Woodgett JR: Multiple phosphoinositide 3-kinase-dependent steps in activation of protein kinase B. Mol Cell Biol 2000; 22: 6247-6260.

17 Rychlik W, Domier LL, Gardner PR, Hellmann GM, Rhoads RE: Amino acid sequence of the mRNA cap-binding protein from human tissues. Proc Nat Acad Sci USA 1987; 84: 945-949.

18 Coelho CM, Leevers SJ: Do growth and cell division rates determine cell size in multicellular organisms? J Cell Sci 2000; 113: $2927-2934$.
19 Montagne J, Stewart MJ, Stocker H, Hafen E, Kozma SC, Thomas G: Drosophila S6 kinase: a regulator of cell size. Science 1999; 285: 2126-2129.

20 Maehama T, Dixon JE: The tumor suppressor, PTEN/MMAC1, dephosphorylates the lipid second messenger, phosphatidylinositol 3,4,5- trisphosphate. J Biol Chem 1998; 273: 13375-13378.

21 Wu X, Senechal K, Neshat MS, Whang YE, Sawyers CL: The PTEN/ MMAC1 tumor suppressor phosphatase functions as a negative regulator of the phosphoinositide 3-kinase/Akt pathway. Proc Natl Acad Sci USA 1998; 95: 15587-15591.

22 Huang $\mathrm{H}$, Potter CJ, Tao W et al: PTEN affects cell size, cell proliferation and apoptosis during Drosophila eye development. Development 1999; 126: 5365-5372.

23 Gao X, Pan D: TSC1 and TSC2 tumor suppressors antagonize insulin signaling in cell growth. Genes Dev 2001; 15: 1383-1392.

24 Potter CJ, Huang H, Xu T: Drosophila Tsc1 functions with Tsc2 to antagonize insulin signaling in regulating cell growth, cell proliferation, and organ size. Cell 2001; 105: 357-368.

25 Shioi T, Kang PM, Douglas PS et al: The conserved phosphoinositide 3-kinase pathway determines heart size in mice. EMBO J 2000; 19: 2537-2548.

26 Shioi T, McMullen JR, Kang PM et al: Akt/protein kinase B promotes organ growth in transgenic mice. Mol Cell Biol 2002; 22: 2799-2809.

27 Kwon $\mathrm{CH}$, Zhu X, Zhang J et al: Pten regulates neuronal soma size: a mouse model of Lhermitte-Duclos disease. Nat Genet 2001; 29: 404-411.

28 Backman SA, Stambolic V, Suzuki A et al: Deletion of Pten in mouse brain causes seizures, ataxia and defects in soma size resembling Lhermitte-Duclos disease. Nat Genet 2001; 29: 396-403.

29 Groszer M, Erickson R, Scripture-Adams DD et al: Negative regulation of neural stem/progenitor cell proliferation by the Pten tumor suppressor gene in vivo. Science 2001; 294: 2186-2189.

$30 \mathrm{Xu} \mathrm{Z}$, Stokoe D, Kane LP, Weiss A: The inducible expression of the tumor suppressor gene PTEN promotes apoptosis and decreases cell size by inhibiting the PI3K/Akt pathway in Jurkat T cells. Cell Growth Differ 2002; 13: 285-296.

31 Grønning LM, Cederberg A, Miura N, Enerback S, Tasken K Insulin and TNF alpha induce expression of the forkhead transcription factor gene Foxc2 in 3T3-L1 adipocytes via PI3K and ERK 1/2-dependent pathways. Mol Endocrinol. 2002; 6: 873-883.

32 Kurotaki N, Imaizumi K, Harada $\mathrm{N}$ et al: Haploinsufficiency of NSD1 causes Sotos syndrome. Nat Genet 2002; 30: 365-366.

33 Albrecht S, Haber RM, Goodman JC, Duvic M: Cowden syndrome and Lhermitte-Duclos disease. Cancer 1992; 70: 869-876.

34 Pascual-Castroviejo I, Patron M, Gutierrez M, Carceller F, PascualPascual SI: Tuberous sclerosis associated with histologically confirmed ocular and cerebral tumors. Pediatr Neurol 1995; 13: 172-174.

35 Di Cristofano A, Pesce B, Cordon-Cardo C, Pandolfi PP: Pten is essential for embryonic development and tumour suppression. Nat Genet 1998; 19: 348-355.

36 Suzuki A, de la Pompa JL, Stambolic V et al: High cancer susceptibility and embryonic lethality associated with mutation of the PTEN tumor suppressor gene in mice. Curr Biol 1998; 8: 1169-1178.

37 Rudolph G, Blum WF, Jenne EW et al: Growth hormone (GH) insulin-like growth factors (IGFs), and IGF-binding protein-3 (IGFBP-3) in a child with Proteus syndrome. Am J Med Genet 1994; 50: 204-210.

38 Hoeflich A, Weber MM, Fisch T et al: Insulin-like growth factor binding protein 2 (IGFBP-2) separates hypertrophic and hyperplastic effects of growth hormone (GH)/IGF-I excess on adrenocortical cells in vivo. FASEB J 2002; 16: 1721-1731.

39 Zhou XP, Marsh DJ, Hampel H, Mulliken JB, Gimm O, Eng C: Germline and germline mosaic PTEN mutations associated 
with a Proteus-like syndrome of hemihypertrophy, lower limb asymmetry, arteriovenous malformations and lipomatosis. Hum Mol Genet 2000; 9: 765-768.

40 Zhou X, Hampel $\mathrm{H}$, Thiele $\mathrm{H}$ et al: Association of germline mutation in the PTEN tumour suppressor gene and Proteus and Proteus-like syndromes. Lancet 2001; 358: 210-211.

41 Smith JM, Kirk EP, Theodosopoulos G et al: Germline mutation of the tumour suppressor PTEN in Proteus syndrome. J Med Genet 2002; 39: 937-940.

42 Barker K, Martinez A, Wang $\mathrm{R}$ et al: PTEN mutations are uncommon in Proteus syndrome. J Med Genet 2001; 38: 480-481.
43 Biesecker LG, Rosenberg MJ, Vacha S, Turner JT, Cohen MM: PTEN mutations and proteus syndrome. Lancet 2001; 358: 2079-2080.

44 Happle R: Elattoproteus syndrome: delineation of an inverse form of Proteus syndrome. Am J Med Genet 1999; 84: 25-28.

45 Wang Y, Spatz MK, Kannan $\mathrm{K}$ et al: A mouse model for achondroplasia produced by targeting fibroblast growth factor receptor 3. Proc Natl Acad Sci USA 1999; 96: 4455-4460.

46 Jonkman MF, Scheffer H, Stulp R et al: Revertant mosaicism in epidermolysis bullosa caused by mitotic gene conversion. Cell 1997; 88: 543-551. 